\title{
Multi-feature Statistical Nonrigid Registration Using High-Dimensional Generalized Information Measures
}

\author{
Sameh Hamrouni ${ }^{1}$, Nicolas Rougon ${ }^{1}$, and Françoise Prêteux ${ }^{2}$ \\ 1 ARTEMIS Department; CNRS UMR 8145 - TELECOM SudParis, Evry, France \\ \{Sameh.Hamrouni, Nicolas.Rougon\} @it-sudparis.eu \\ 2 Direction des Recherches - Mines ParisTech, Paris, France
}

\begin{abstract}
Nonrigid image registration methods based on the optimization of information-theoretic measures provide versatile solutions for robustly aligning mono-modal data with nonlinear variations and multi-modal data in radiology. Whereas mutual information and its variations arise as a first choice, generalized information measures offer relevant alternatives in specific clinical contexts. Their usual application setting is the alignement of image pairs by statistically matching scalar random variables (generally, greylevel distributions), handled via their probability densities. In this paper, we address the issue of estimating and optimizing generalized information measures over high-dimensional state spaces to derive multi-feature statistical nonrigid registration models. Specifically, we introduce novel consistent and asymptotically unbiaised $k$ nearest neighbors estimators of $\alpha$-informations, and study their variational optimization over finite and infinite dimensional smooth transform spaces. The resulting theoretical framework provides a well-posed and computationally efficient alternative to entropic graph techniques. Its performances are assessed on two cardiological applications: measuring myocardial deformations in tagged MRI, and compensating cardio-thoracic motions in perfusion MRI.
\end{abstract}

Keywords: Multi-feature nonrigid registration, groupwise nonrigid registration, kNN entropy estimators, high-dimensional $\alpha$-information, cardiac tagged MRI, cardiac perfusion MRI.

\section{Introduction}

Nonrigid image registration methods based on the optimization of information measures provide versatile solutions for robustly aligning mono-modal data with nonlinear variations and multi-modal (inhomogeneous or incommensurable) data in medical radiology. Whereas mutual information and its variations arise as a first choice [8], generalized information measures, such as $f$-informations [1112] and Jensen $f$-divergences [9], have been shown to be relevant alternatives in specific clinical contexts.

Their usual application setting is the alignement of image pairs by statistically matching luminance distributions, handled using marginal and joint probability densities estimated via kernel techniques. Though efficient for joint densities exhibiting well-separated clusters or reducible to simple mixtures, these approaches reach their limits for nonlinear mixtures, such as generated by textured objects over complex backgrounds. In this

G. Fichtinger, A. Martel, and T. Peters (Eds.): MICCAI 2011, Part I, LNCS 6891, pp. 524-531, 2011.

(c) Springer-Verlag Berlin Heidelberg 2011 
case, pixelwise luminance appears to be a too coarse feature for allowing unambiguous local statistical decisions, resulting into inaccurate or inconsistent matches, especially problematic in atlas propagation or motion estimation frameworks.

An intuitive idea to overcome this limitation is to combine multiple higher-dimensional image features (which may convey spatial information) within a vector random variable (RV). Its implementation, however, is faced with the curse of dimensionality: the number of samples required to consistently estimate a probability density increases exponentially with the state space dimension. In particular, kernel density estimators fail in high dimensions due to the fixed size of sample sets, which precludes estimating and optimizing information measures using plug-in techniques. Groupwise registration of image series, i.e. their simultaneous alignment onto a common reference, comes up against a similar obstacle, linked to handling spatio-temporal luminance distributions.

This theorerical issue can be bypassed by computing information measures directly from data using geometric entropy estimators. The latter include (i) entropic graphs, applicable to the broader class of $\alpha$-informations, whose relevance for image registration is today well-established [9]13]; and (ii) more recent $k$ th-nearest neighbor $(\mathrm{kNN})$ entropy estimators [4], whose application to image science has been until today confined to the Shannon framework [35]6].

In this paper, we introduce novel, consistent and asymptotically unbiased kNN estimators of $\alpha$-informations, and study their variational optimization over finite- and infinite-dimensional spaces of smooth spatial mappings. The resulting theoretical framework provides a well-posed and computationally efficient alternative to entropic graph techniques for multi-feature nonrigid image registration. Its performances are assessed in cardiac MRI for two clinical challenges: measuring myocardial deformations in tagged MRI, and compensating cardio-thoracic motions in perfusion MRI.

\section{Multi-feature Information-Theoretic Registration}

\subsection{Problem Statement and Modeling}

In order to build a unified formalism including pairwise and groupwise approaches, we deal with the general issue of registering synchronized image sequences. A sequence of 2D greylevel images defined over a domain $\Omega \subset \mathbb{R}^{2}$ is modeled as a mapping $I: \Omega \times$ $[1 . . d] \rightarrow \Lambda$ with values in an interval $\Lambda \subset \mathbb{Z}$. Given a source sequence $I_{M}$ comprising $d$ unregistered images, and a reference sequence $I_{T}$ with the same length, let $x_{\tau}$ denote the spatial coordinates 1 in frame $I_{M}(\cdot, \tau)$ and let $x=\left[x_{1} \ldots x_{d}\right] \in \Omega^{d}$. We seek for a $d$-dimensional vector of smooth spatial transforms $\phi(x)=\left[\phi_{1}\left(x_{1}\right) \ldots \phi_{d}\left(x_{d}\right)\right]$ in some space $\mathcal{T}$ such that $\phi_{\tau}(\tau \in[1 . . d])$ maps the source frame $I_{M}(\cdot, \tau)$ onto its analog $I_{T}(\cdot, \tau)$ in the reference sequence. The case $d=1$ amounts to pairwise registration. Groupwise registration corresponds to $d>1, I_{T}$ being either a registered sequence or the concatenation of $d$ instances of the same reference image.

Local correspondences are established via a dense set $M$ (resp. $T$ ) of spatio-temporal features extracted from $I_{M}\left(I_{T}\right)$ with values in a $D$-dimensional space $\mathcal{F}$, which can comprise both radiometric and spatial information. Features are integrated in vectors

\footnotetext{
${ }^{1}$ Similarly, the $\tau$ th component of a vector $r$ is hereafter denoted by $r_{\tau}$.
} 
$M(x)=\left[M\left(x_{1}, 1\right) \ldots M\left(x_{d}, d\right)\right] \in \mathcal{F}$ (resp. $\left.T(x)\right)^{2}$. To robustly account for sensor-, patient- and pathology-related variability, we adopt a statistical framework, and model $M$ and $T$ as random variables $(\mathrm{RV})$ over the state space $\mathcal{F}$, with respective marginal densities $p^{M}$ and $p^{T}$, joint density $p^{M, T}$ and copula density $\pi^{M, T}=\frac{p^{M, T}}{p^{M} p^{T}}$.

Registration is then tackled as an optimal statistical matching problem between feature distributions over the transform space $\mathcal{T}: \phi^{*}=\operatorname{argmin}_{\phi \in \mathcal{T}} \mathcal{C}(M, T, \phi)$. The matching criterion $\mathcal{C}(M, T, \phi)$ is expressed as the sum of a discrepancy term $\mathcal{S}\left(M^{\phi}, T\right)$ between the reference RV $T$ and the RV $M^{\phi}$ computed from the warped sequence $I_{M}^{\phi}=I_{M} \circ \phi^{-1}$, shortly denoted by $M$; and a regularization term $\mathcal{R}(\phi)$ ensuring smooth solutions. In this paper, we study the $\alpha$-information family, comprising the pseudo-additive Havrda-Charvát information $I_{\alpha}(M, T)=\frac{1}{\alpha-1}\left(J_{\alpha}(M, T)-1\right)$ and the additive Renyi information $I_{\alpha}^{*}(M, T)=\frac{1}{\alpha-1} \log J_{\alpha}(M, T)$, where:

$$
J_{\alpha}(M, T)=\int_{\mathcal{F}^{2}} p^{M, T}(m, t)\left(\pi^{M, T}(m, t)\right)^{\alpha-1} d m d t=\mathbb{E}_{M, T}\left[\left(\pi^{M, T}\right)^{\alpha-1}\right]
$$

These measures, defined for $\alpha \in \mathbb{R}^{+} \backslash\{0,1\}$, generalize mutual information which is obtained in the limit $\alpha \rightarrow 1$, yielding discrepancy functionals $\mathcal{S}=-I_{\alpha}$ and $\mathcal{S}=-I_{\alpha}^{*}$.

\section{$2.2 \mathrm{kNN}$ Estimators of $\alpha$-Informations}

Given a $q$-dimensional RV $Y$, let $Y\left(\Omega^{d}\right)$ be a set of i.i.d. samples $Y(x)$ of $Y$ indexed by $x \in \Omega^{d}$. kNN estimation techniques aim at characterizing $Y$ from the statistics of $q$-dimensional balls $\mathcal{B}_{k}^{Y}(x) \subset \mathbb{R}^{q}$, centered at sample $Y(x)$ and containing its $k$ nearest neighbors in $Y\left(\Omega^{d}\right) \backslash\{Y(x)\}$. The resulting estimators are functions of the radius $\rho_{k}^{Y}(x)$ of $\mathcal{B}_{k}^{Y}(x)$, equal to the Euclidean distance from $Y(x)$ to its $k$ th nearest neighbor in this set. An early example is the Loftsgaarden-Quesenberry density estimator: $p_{\mathrm{LQ}}^{Y}(Y(x))=\frac{k}{|\Omega| V_{q}\left(\rho_{k}^{Y}(x)\right)^{q}}$. Though notoriously biased in high dimension, the latter leads however to a consistent and asymptotically unbiased kNN estimator of Shannon entropy [4], from which kNN estimators of Kullback-Leibler divergence [4]], and mutual information and its variations [5]6] have been derived.

This result has been recently extented to $\alpha$-entropies [7], based on a consistent and asymptotically unbiased kNN estimator of the integral $J_{\alpha}(Y)=\int_{\mathbb{R}^{q}}\left(p^{Y}(y)\right)^{\alpha} d y$ :

$$
J_{\alpha}^{\mathrm{knn}}(Y)=\frac{1}{|\Omega|} \sum_{x \in \Omega^{d}}\left((|\Omega|-1) C_{k}(\alpha) V_{q}\left[\rho_{k}^{Y}(x)\right]^{q}\right)^{1-\alpha}
$$

where $C_{k}(\alpha)=\left[\frac{\Gamma(k)}{\Gamma(k+1-\alpha)}\right]^{\frac{1}{1-\alpha}}$ and $V_{q}=\frac{\pi^{q / 2}}{\Gamma(q / 2+1)}$ is the volume of the unit ball of $\mathbb{R}^{q} . J_{\alpha}^{\mathrm{knn}}(Y)$ can be interpreted as a plug-in estimator, built from an Ahmad-Lin estimator [1] $J_{\alpha}^{\mathrm{AL}}(Y)=\frac{1}{|\Omega|} \sum_{x \in \Omega^{d}}\left(p^{Y}(Y(x))\right)^{\alpha-1}$ of $J_{\alpha}(Y)=\mathbb{E}_{Y}\left[\left(p^{Y}\right)^{\alpha-1}\right]$ using the following kNN density estimator:

$$
p_{\mathrm{knn}}^{Y}(Y(x))=\frac{1}{(|\Omega|-1) C_{k}(\alpha) V_{q}\left(\rho_{k}^{Y}(x)\right)^{q}}
$$

\footnotetext{
${ }^{2}$ Here, $M\left(x_{\tau}, \tau\right)$ is a $D_{1}$-dimensional feature vector extracted from $I_{M}(\cdot, \tau)$ with $D=d D_{1}$.
} 
Along the same lines, we derive high-dimensional kNN estimators of $\alpha$-informations by introducing a novel consistent and asymptotically unbiased estimator of the integral $J_{\alpha}(M, T)$, defined as:

$$
J_{\alpha}^{\mathrm{knn}}(M, T)=\frac{1}{|\Omega|} \sum_{x \in \Omega^{d}}\left((|\Omega|-1) C_{k}(\alpha) \frac{V_{D}^{2}}{V_{2 D}}\left[\frac{\rho_{k}^{M}(x) \rho_{k}^{T}(x)}{\left(\rho_{k}^{M, T}(x)\right)^{2}}\right]^{D}\right)^{\alpha-1}
$$

Building this estimator, which is achieved similarly to the entropy estimator $J_{\alpha}^{\operatorname{knn}}(Y)$, is formally equivalent to plugging the kNN copula density estimator $\pi_{\mathrm{knn}}^{M, T}=\frac{p_{\mathrm{knn}}^{M, T}}{p_{\mathrm{knn}}^{M} p_{\mathrm{knn}}^{T}}$ of $\pi^{M, T}$ in the following Ahmad-Lin estimator of $J_{\alpha}(M, T)$ :

$$
J_{\alpha}^{\mathrm{AL}}(M, T)=\frac{1}{|\Omega|} \sum_{x \in \Omega^{d}}\left(\pi^{M, T}(x)\right)^{\alpha-1}
$$

where $\pi^{M, T}(x)=\pi^{M, T}(M(x), T(x))$. From (2), we obtain consistent estimators $I_{\alpha}^{\mathrm{knn}}(M, T)=\frac{1}{\alpha-1}\left(J_{\alpha}^{\mathrm{knn}}(M, T)-1\right)$ and $I_{\alpha}^{* \mathrm{knn}}(M, T)=\frac{1}{\alpha-1} \log J_{\alpha}^{\mathrm{knn}}(M, T)$ of Havrda-Charvát and Renyi information, respectively. In addition, $I_{\alpha}^{\mathrm{knn}}(M, T)$ is asymptotically unbiased.

\subsection{Variational Optimization}

Searching for the optimal transform $\phi^{*}$ using gradient descent requires a closed-form expression of the first variation of $\alpha$-informations over the transform space $\mathcal{T}$. Since the kNN estimator $J_{\alpha}^{\mathrm{knn}}(M, T)$ is not differentiable, a two-step plug-in approach is used. Starting from consistent Ahmad-Lin estimators $I_{\alpha}^{\mathrm{AL}}(M, T)=\frac{1}{\alpha-1}\left(J_{\alpha}^{\mathrm{AL}}(M, T)-1\right)$ and $I_{\alpha}^{* \mathrm{AL}}(M, T)=\frac{1}{\alpha-1} \log J_{\alpha}^{\mathrm{AL}}(M, T)$, we first classically compute a closed-form expression of their variational derivatives using differentiable kernel density estimates and a Mean-Shift approximation of the ratio $\nabla p / p$ for the densities $p^{M}$ and $p^{M, T}$. We then switch to the kNN framework by considering uniform kernels over the balls $\mathcal{B}_{k}^{M}$ and $\mathcal{B}_{k}^{M, T}$ (their derivatives being indicators over the spheres $\mathcal{S}_{k}^{M}$ and $\mathcal{S}_{k}^{M, T}$ ). This strategy yields consistent kNN estimators of the variational derivative of $\alpha$-informations.

For infinite dimensional transform spaces, consisting of smooth mappings $\phi_{\tau}=$ $\mathbb{I d}+u_{\tau}$ described by displacement fields $u_{\tau}$ to be estimated at all $x \in \Omega^{d}$, we get:

$$
\partial_{u_{\tau}} J_{\alpha}^{\mathrm{knn}}(x)=\mathcal{V}(x) \mathcal{D}_{\tau}^{M}(x)
$$

where $\mathcal{V}(x)=c_{3}\left[K_{k}^{M, T}(x)\left(\mathcal{L}_{k, \tau}^{M}(x)-\mathcal{L}_{k, \tau}^{M, T}(x)\right)+c_{2}\left(L_{k, \tau}^{M}(x)-L_{k, \tau}^{M, T}(x)\right)\right]$ and $\mathcal{D}_{\tau}^{M}(x)=\left(\partial_{x_{\tau}} M(x)\right)^{T}$. Letting $\mathcal{N}_{k}^{M, T}(x)=\left\{y \in \Omega^{d} \mid x \in \mathcal{S}_{k}^{M, T}(y)\right\}$, we have:

$$
\begin{aligned}
\mathcal{L}_{k, \tau}^{M, T}(x) & =\frac{2 d+2}{\rho_{k}^{M, T}(x)^{2}} \sum_{y \in \mathcal{B}_{k}^{M, T}(x)} r^{M}(x, y) & L_{k, \tau}^{M, T}(x) & =\sum_{y \in \mathcal{N}_{k}^{M, T}(x)} \frac{r^{M}(x, y)}{\left|r^{M}(x, y)\right|}\left(\pi_{\mathrm{knn}}^{M, T}(y)\right)^{\alpha-1} \\
\mathcal{L}_{k, \tau}^{M}(x) & =\frac{d+2}{\rho_{k}^{M}(x)^{2}} \sum_{y \in \mathcal{B}_{k}^{M}(x)} r^{M}(x, y) & L_{k, \tau}^{M}(x) & =\sum_{y \in \mathcal{N}_{k}^{M}(x)} \frac{r^{M}(x, y)}{\rho_{k}^{M}(y)}\left(\pi_{\mathrm{knn}}^{M, T}(y)\right)^{\alpha-1}
\end{aligned}
$$




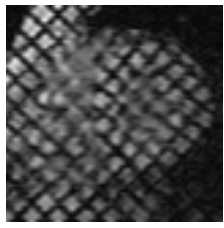

$I_{M}$

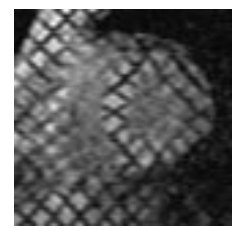

$I_{T}$

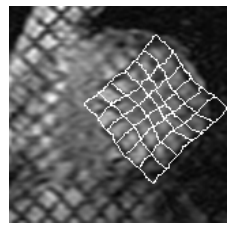

$I_{M}^{\mathbf{u}^{*}}$

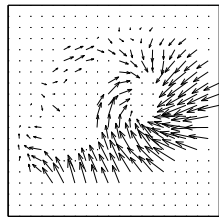

$\mathbf{u}^{*}=\phi^{*}-\mathbb{I d}$

Fig. 1. Estimating myocardial deformations in tagged MRI using multi-feature Havrda-Charvát information-minimizing nonrigid registration

Finally: $K_{k}^{M, T}(x)=\frac{1}{k}\left(\pi_{\mathrm{knn}}^{M, T}(x)\right)^{\alpha-1}, c_{2}=C_{k}(\alpha) \frac{|\Omega|-1}{|\Omega|}$ and $c_{3}=\frac{\alpha-1}{|\Omega|}$.

The optimal transforms $\phi_{\tau}^{*}$ are iteratively estimated at every pixel $x \in \Omega^{d}$ with the explicit scheme: $u_{\tau}^{t+1}(x)=u_{\tau}^{t}(x)+\delta_{t} \partial_{u_{\tau}} I_{\alpha}{ }^{\mathrm{knn}}(x)$ and accordingly for $I_{\alpha}^{* \mathrm{knn}}$.

For finite dimensional transform spaces, consisting of smooth mappings $\phi_{\tau}\left(x_{\tau}\right)=$ $B\left(x_{\tau}\right) \Theta_{\tau}$ described by a parameter $\Theta_{\tau} \in \mathbb{R}^{p}$ in a local basis encoded by the $(2 \times p)$ matrix $B\left(x_{\tau}\right)$, we get:

$$
\partial_{\Theta_{\tau}} J_{\alpha}^{\mathrm{knn}}=\sum_{x \in \Omega^{d}} \mathcal{V}(x) \mathcal{D}_{B, \tau}^{M}(x)
$$

where $\mathcal{D}_{B, \tau}^{M}(x)=\left(\partial_{x_{\tau}} M(x)\right)^{T} B\left(x_{\tau}\right)$. The optimal parameters $\Theta_{\tau}^{*}$ are computed using the update equation: $\Theta_{\tau}^{t+1}=\Theta_{\tau}^{t}+\delta_{t} \partial_{\Theta_{\tau}} I_{\alpha}{ }^{\mathrm{knn}}\left(\Theta^{t}\right)$ and accordingly for $I_{\alpha}^{* \mathrm{knn}}$.

\subsection{Implementation Details}

The numerical complexity of the optimization scheme, dictated by the kNN search algorithm, is reduced by using an approximate nearest neighbor technique [2] with linear complexity and memory usage w.r.t. the number of samples $|\Omega|$ and feature dimension $D$. Obviously, complexity increases with $k$. In practise, this parameter is tuned empirically by searching for a trade-off between registration accuracy and processing time. For the cardiac MRI applications hereafter, setting $k=15$ yielded satisfying results.

\section{Application to Cardiac MRI}

\subsection{Estimating Myocardial Motion in Tagged MRI Using Pairwise Registration}

The proposed model has been applied to the estimation of myocardial deformations from tagged MRI exams by sequentially performing frame-to-frame registration [12]. We used a local feature vector with normalized components, comprising the greylevel, and the eigenvalues and eigenvectors of the structure tensor $\partial_{x_{\tau}} I(x)^{T} \partial_{x_{\tau}} I(x) \star G_{\sigma}$ of the image $I$, computed at a Gaussian scale $\sigma$ equal to the half-width of the tagging pattern $(D=5)$. We studied the impact on registration accuracy of using a directional local contrast descriptor by comparing performances with a similar registration model exploiting solely the greylevel information [12]. To be consistent with the later work, we considered an infinite dimensional (non-parametric) transform space, and a registration 
Table 1. MSE (in pixels) between estimated and ground-truth motion magnitude for simulated tagged MRI datasets using greylevel $(D=1)$ and greylevel + structure tensor $(D=5)$ features

\begin{tabular}{c|ccc}
$D$ & Base & Median & Apex \\
\hline \hline 1 & $0.73 \pm 0.31$ & $0.88 \pm 0.51$ & $1.53 \pm 0.75$ \\
5 & $0.35 \pm 0.22$ & $0.42 \pm 0.34$ & $0.65 \pm 0.59$ \\
\hline
\end{tabular}

Table 2. MSE and Pearson correlation coefficient $\left(R^{2}\right)$ between estimated and ground-truth parameters as a function of maximal displacement $\delta$ (in pixels) for simulated p-MRI datasets at median level with random FFD misalignment

\begin{tabular}{cr|ccccc} 
& $\delta$ & 1 & 3 & 5 & 7 & 10 \\
\hline \hline \multirow{2}{*}{ MSE } & NMI & 0.52 & 0.76 & 0.63 & 0.83 & 1.5 \\
& $I_{\alpha}^{*}$ & 0.41 & 0.49 & 0.57 & 0.62 & 0.80 \\
\cline { 2 - 7 }$R^{2}$ & NMI & 0.94 & 0.91 & 0.93 & 0.90 & 0.83 \\
& $I_{\alpha}^{*}$ & 0.95 & 0.94 & 0.93 & 0.93 & 0.91 \\
\hline
\end{tabular}

Table 3. Dice similarity indices between reference and registration-induced segmentations of the left ventricle (Lv), right ventricle ( $\mathrm{Rv})$ and myocardium (Myo)

\begin{tabular}{r|ccc} 
& $\mathrm{Lv}$ & $\mathrm{Rv}$ & Myo \\
\hline \hline native & 0.89 & 0.003 & 0.20 \\
registered using NMI & 0.98 & 0.95 & 0.88 \\
registered using $I_{\alpha}^{*}$ & 0.98 & 0.87 & 0.93 \\
\hline
\end{tabular}

criterion combining Havrda-Charvát information $(\alpha=1.2)$ and the Nagel-Enkelmann oriented smoothness stabilizer, and performed experiments on the SPAMM dataset used in [12]. Qualitative assessment of the estimated displacement fields by an expert cardiologist indicate systematic improvement of motion estimates (i) on endo- and epicardial boundaries, where the tagging signal suffers from poor localization and contrast fading artifacts, and (ii) over the whole myocardium at end of sequence (Figure 1). This was confirmed on synthetic tagged MRI sequences generated from natural exams by warping their first frame with the statistical myocardial motion atlas described in [10]. Comparing the MSE between ground-truth and estimated motion over the dataset shows that accuracy is improved by a multi-feature registration model (Table 1 ).

\subsection{Groupwise Registration of Perfusion MRI Exams}

The proposed model has been also applied to the compensation of cardio-thoracic motions in partial breath-hold and free-breathing contrast-enhanced cardiac perfusion MRI (p-MRI) exams. Here, the challenge lies in aligning structures exhibiting highly nonlinear contrast variations and complex deformations. We adopt a groupwise registration strategy involving a motion-free reference $\mathrm{p}$-MRI exam and using pixel-wise contrast enhancement curves $M(x)=\left[I_{M}\left(x_{1}, 1\right) \ldots I_{M}\left(x_{d}, d\right)\right]$ (resp. $T(x)$ ) as local features ( $D=d \in[25 . .35])$. Using spatio-temporal features induces an alignment process driven by the statistical properties of the whole exam, with the expected benefit of an improved alignment overall consistency compared to classical pairwise schemes based 

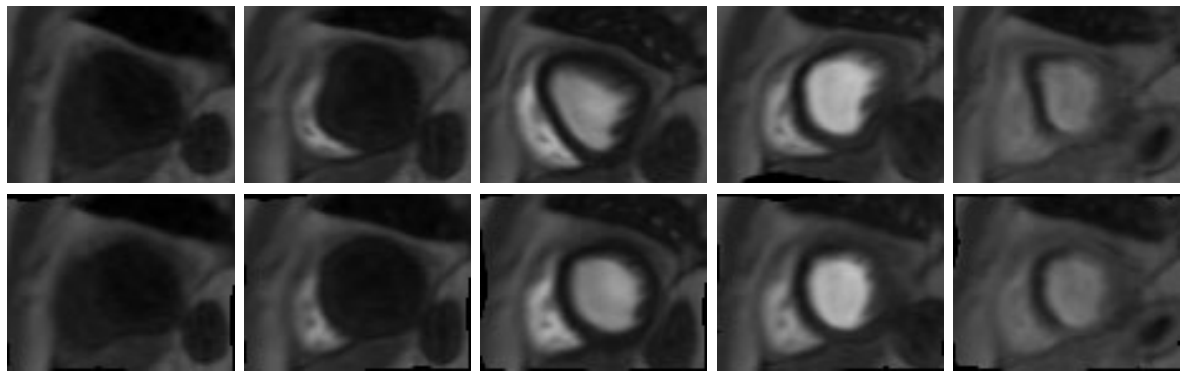

Fig. 2. Evaluation of registration accuracy. Top: Simulated unregistered exam $(\delta=7)$ at median level $\left(d=34\right.$ frames). Bottom: registration over a reference motion-free exam $I_{T}$ using $I_{\alpha}^{*}$.
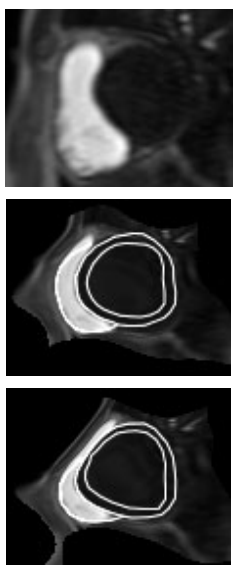
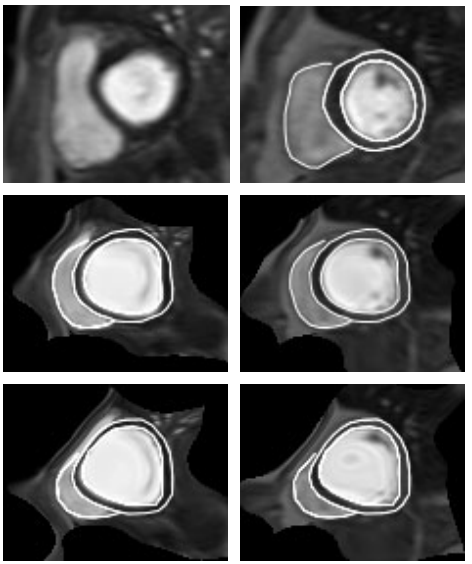
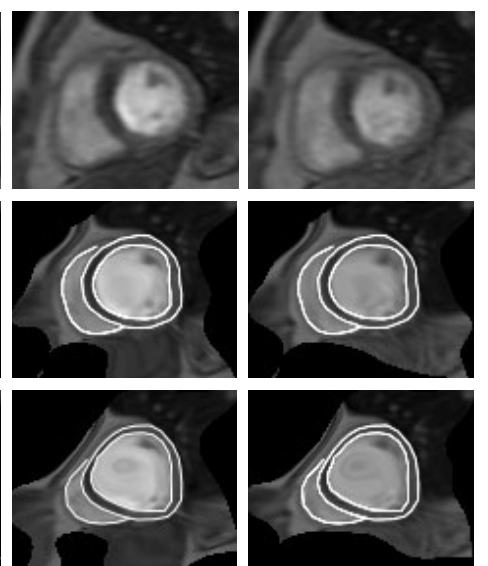

Fig. 3. Evaluation of registration robustness. Top: unregistered synthetic exam at median level created by concatenating $3+3$ frames from 2 different patient exams. Middle (resp. bottom): registration using NMI (resp. $I_{\alpha}^{*}$ ). Overlay: registration-induced segmentation derived from a supervised delineation of the heart in a reference frame (top) replicated on all images. Warping artefacts over thoracic regions originate from using a synthetic template with uniform background.

on a reference frame with maximal myocardial contrast. We use a matching criterion combining Renyi information $(\alpha=1.2)$ and a thin-plate spline stabilizer, which is optimized over a Free-Form Deformation (FFD) basis parameterized by $8 \times 8$ control grids. Registration results were systematically compared with those delivered by a similar model [6] using a high-dimensional kNN estimator of normalized mutual information (NMI) instead of Renyi information. To this end, the dataset and experimental validation protocol described in [6] were used. Results on synthetic sequences, generated by applying to the reference exam random FFD transforms with control point displacements uniformly distributed in the $[-\delta, \delta]$ pixels range (Figure 2), show that Renyi information leads to improving registration accuracy (Table 2). Similar conclusions were reached for registration robustness (Table 3), which was assessed on composite datasets synthetized by intermixing frames from different patient exams at the same slice level 
(Figure 3). Processing $d=35$ frames at 3 slice levels (i.e. base, medium, apex) requires approximately 13 minutes on a standard $2.4 \mathrm{GHz}$ dual core PC with $2 \mathrm{~Gb}$ RAM.

\section{Conclusion}

We have presented novel kNN estimators of high-dimensional $\alpha$-informations, and derived closed-form expressions of their first variation over finite- and infinite-dimensional spaces of smooth spatial tranforms. This theoretical framework enables variational multifeature nonrigid registration based on a non-Shannon information-theoretic model, and provides a computationally efficient alternative to entropic graph techniques. The formalism, developed for the alignement of 2D image sequences, encompasses both pairwise and groupwise registration, and is obviously readily valid in 3D. Future work is currently directed towards increasing computational performances by using more efficient gradient descent schemes and boosting $\mathrm{kNN}$ search using GPU implementations.

\section{References}

1. Ahmad, I., Lin, P.: A nonparametric estimation of the entropy for absolutely continuous distributions. IEEE Transactions on Information Theory 22(3), 372-375 (1976)

2. Arya, S., Mount, D.M., Netanyahu, N.S., Silverman, R., Wu, A.: An optimal algorithm for approximate nearest neighbor searching. Journal of the ACM 45(6), 891-923 (1998)

3. Boltz, S., Debreuve, E., Barlaud, M.: High-dimensional statistical measure for region-ofinterest tracking. IEEE Transactions on Image Processing 18(6), 1266-1283 (2009)

4. Goria, M., Leonenko, N., Mergel, V., Novi Inverardi, P.L.: A new class of random vector entropy estimators and its applications in testing statistical hypotheses. Journal of Nonparametric Statistics 17(3), 277-297 (2005)

5. Hamrouni, S., Rougon, N., Prêteux, F.: Multi-feature information-theoretic image registration: application to groupwise registration of perfusion MRI exams. In: Proceedings IEEE International Symposium on Biomedical Imaging: Fron Nano to Macro, Chicago, IL (2011)

6. Hamrouni, S., Rougon, N., Prêteux, F.: Groupwise registration of cardiac perfusion MRI sequences using normalized mutual information in high dimension. In: Proceedings SPIE Medical Imaging 2011 - Image Processing, Orlando, FL, vol. 7962 (2011)

7. Leonenko, N., Pronzato, L., Savani, V.: A class of Rényi information estimators for multidimensional densities. Annals of Statistics 36(5), 2153-2182 (2008)

8. Maes, F., Vandermeulen, D., Suetens, P.: Medical image registration using mutual information. Proceedings of the IEEE 91(10), 1699-1722 (2003)

9. Neemuchwala, H.F., Hero, A.O.: Entropic graphs for registration. In: Multi-sensor Image Fusion and its Applications. Marcel Dekker, New York (2004)

10. Petitjean, C., Rougon, N., Prêteux, F.: Building and using a statistical 3D motion atlas for analyzing myocardial contraction in MRI. In: Proceedings SPIE Medical Imaging 2004 Image Processing, San Diego, CA, vol. 5370, pp. 253-264 (2004)

11. Pluim, J.P.W., Maintz, J.B.A., Viergever, M.A.: $f$-information measures in medical image registration. IEEE Transactions on Medical Imaging 23(12), 1508-1516 (2004)

12. Rougon, N., Petitjean, C., Prêteux, F., Cluzel, P., Grenier, P.: A non-rigid registration approach for quantifying myocardial contraction in tagged MRI using generalized information measures. Medical Image Analysis 9(4), 353-375 (2005)

13. Staring, M., van der Heide, U.A., Klein, S., Viergever, M.A., Pluim, J.P.W.: Registration of cervical MRI using multifeature mutual information. IEEE Transactions on Medical Imaging 28(9), 1412-1421 (2009) 